\title{
El valor económico de la vacuna contra el dengue: un estudio de valoración contingente*
}

Economic Value of the Dengue
Vaccine: A Contingent Valuation Study
O valor econômico da vacina contra a
dengue: estudo de valoração contingente
Fecha de recepción: 27 Septiembre 2018. Fecha de aprobación:
15 Mayo 2019. Fecha de publicación: 29 Noviembre 2019.
DOI: https://doi.org/10.11144/Javeriana.rgps18-37.vevc
Kocfa Chung-Delgado ${ }^{\text {a }}$
Universidad Peruana de Ciencias Aplicadas, Perú
ORCID: http://orcid.org/0000-0003-1962-9434
Laura Navarro-Huaman
Universidad Peruana de Ciencias Aplicadas, Perú
ORCID: http://orcid.org/0000-0002-1703-3912

Cómo citar este artículo:Chung-Delgado K, Navarro-Huaman L. El valor económico de la vacuna contra el dengue: un estudio de valoración contingente. Revista Gerencia y Políticas de Salud. 2019;18(37). https://doi.org/10.11144/Javeriana.rgps18-37.vevc

\footnotetext{
${ }^{\mathrm{a}}$ Autor de correspondencia. Correo electrónico: kocfachung@gmail.com
} 


\section{Resumen}

El dengue ha presentado rápido crecimiento a nivel mundial. El objetivo de este estudio fue determinar la disponibilidad a pagar (DAP) para valorar económicamente el acceso a una vacuna contra dengue y sus factores asociados. Se ejecutó un estudio de valoración contingente de tipo trasversal, que evaluó la DAP y factores asociados en la población de Piura, Perú. Se empleó un cuestionario heteroaplicado de formato binario-dicotómico doble de elección discreta, en modalidad de entrevista personal. Se incluyeron 371 individuos en el análisis. La mediana de DAP máxima fue S/. 210 (Nuevo Sol Peruano, PEN). Las variables asociadas a una compra de la vacuna fueron ser profesional de salud, educación superior y antecedente personal y familiar de dengue. La definición del precio es un elemento clave para el desarrollo y parametrización de este nuevo mercado. Autoridades sanitarias deben tomar en consideración la evidencia económica disponible para diseñar intervenciones sostenibles para esta nueva tecnología.

Palabras clave: vacunas contra dengue, dengue, vacunas, análisis costo-beneficio, economía de la salud, economía médica.

\section{Abstract}

Dengue has shown a fast growth worldwide. Therefore, this study aims to determine the Money Availability (DAP, in Spanish) in order to do a financial valuation of the people's access to a dengue vaccine and the related factors. A contingent valuation cross-sectional study was conducted in order to assess the Money Availability and the related factors in the population of Piura, Perú. A hetero-applied questionnaire with a double binary-dichotomic format and discrete choice was used; it was applied in the mode of a personal interview. The analysis included 371 individuals. The highest Money Availability was S/. 210 (New Peruvian Sol, PEN). The variables associated with buying the vaccine were working as a health professional, having earned a college degree, having personal or family background of dengue. The vaccine pricing is a key factor in the development and standardization of this new market. The public health authorities must consider the financial evidence thus available when designing sustainable interventions for this new technology.

Keywords: dengue vaccine, dengue, vaccines, cost-benefit analysis, health economy, medical economy.

\section{Resumo}

A dengue já mostrou rápido crescimento no nível mundial. O objetivo deste estudo foi determinar a disponibilidade a pagar (DAP) para avaliar economicamente o acesso à vacina contra a dengue e seus fatores associados. Desenvolveu-se estudo de valoração contingente de tipo transversal que avaliou a DAP e fatores associados na população de Piura, Peru. Foi usado inquérito heteroaplicado de formato binário-dicotómico duplo de escolha discreta, na forma de entrevista pessoal. Incluíram-se 371 indivíduos na análise. A mediana de DAP máxima foi S/210 (Nuevo Sol Peruano, PEN). As variáveis associadas a uma compra da vacina foram ser profissional da saúde, ensino superior e histórico pessoal e familiar de dengue. A definição do preço é um elemento chave para o desenvolvimento e parametrização deste novo mercado. Autoridades sanitárias devem levar em consideração a evidência econômica disponível para o desenho de intervenções sustentáveis para essa nova tecnologia.

Palavras-chave: vacinas contra a dengue, dengue, vacinas, análise custo-benefício, economia da saúde, economia médica. 


\section{Introducción}

En el año 2015 se anunció la primera aprobación regulatoria de una vacuna contra el dengue (1). Según la Organización Mundial de la Salud (OMS), el dengue es la enfermedad transmitida por mosquitos de más rápido crecimiento a nivel mundial $(2,3)$. La incidencia de esta enfermedad se ha incrementado por un factor de 30, donde se estiman hasta 100 millones de nuevas infecciones anuales en más de 100 países endémicos (2).

La carga económica anual del dengue en el continente Americano ha sido estimada en aproximadamente \$2,1 mil millones (USD \$2010), lo que significa que tiene un costo mediano de $\$ 472$ por caso ambulatorio (4). En el Sudeste asiático, la enfermedad provoca una pérdida de 372 años de vida ajustados por discapacidad (AVAD) por cada millón de habitantes (5). En Perú, los casos de dengue siguen la misma tendencia en incremento (6). El último brote epidemiológico nacional, cerrado a la semana epidemiológica 52 del 2016, notificó 76.093 casos de dengue, de los cuales más del 64,04\% están concentrados en la región de Piura (7).

La eficacia y seguridad de esta vacuna ha sido estudiada a través de investigaciones que incluyen todas las fases de desarrollo clínico (3). Actualmente, la vacuna se encuentra aprobada en 19 países por sus autoridades regulatorias respectivas para su uso local, y se viene introduciendo paulatina y progresivamente en los demás países de mayor carga endémica por dengue en el mundo (1).

La vacuna ha sido reevaluada recientemente por el grupo Sage de la OMS (8), lo cual ha suscitado una importante discusión respecto a la misma. En esta, el precio se identifica como un posible obstáculo para la distribución y acceso en términos de servicio de salud $(9,10)$, donde aún se considera su impacto en términos económicos a nivel mundial $(4,5)$.

Desde el punto de vista de economía de la salud y estudios costo-beneficio, existe evidencia científica limitada que describe el acceso a una vacuna contra dengue $(11,12,13,14,15)$. La mayoría de estos estudios han evaluado la vacuna desde una perspectiva teórica, ya que fueron ejecutados antes de la comercialización global de la misma $(12,13,15)$.

Este aporte científico, tanto desde el punto de vista médico como económico, es de gran soporte para las decisiones en términos de servicios y economía de salud, ya que brinda una perspectiva social de la población en términos de disponibilidad (16). Así mismo, la evidencia de este tipo de estudios es usada cada vez más por países y autoridades sanitarias $(16,17)$.

La vacuna contra dengue, a pesar del número limitado de opciones en la actualidad, ha sido contemplada dentro de los planes estratégicos de control y prevención de la enfermedad de la Organización Mundial de la Salud y la Organización Panamericana de la Salud $(2,18)$, que afirman que "la disponibilidad de una vacuna segura, eficaz y costo-efectiva alteraría significativamente el concepto de prevención contra dengue" (2). Un análisis sólido que integre ambos factores contribuiría al desarrollo y la sostenibilidad de esta nueva estrategia sanitaria 
en el tiempo, tanto para los decisores políticos como para los agentes financiadores (2), considerando que la vacunación es una de las intervenciones de mayor impacto en salud pública y reducción de mortalidad (19).

El objetivo principal de esta investigación es valorar económicamente la disponibilidad a pagar (DAP) de acceso a una vacuna contra dengue, desde la perspectiva de los individuos, en la población general mayor de 18 años proveniente de la provincia de Piura, Perú con ingreso o renta individual mayor de cero. Los objetivos específicos de esta investigación son: 1) definir si el acceso a una vacuna contra dengue supone un beneficio adicional a la salud, 2) determinar la disponibilidad a pagar (DAP) en moneda local para tener acceso a la vacuna contra dengue, y 3) evaluar factores asociados a cambios y variaciones en la DAP.

\section{Materiales y métodos}

Se ejecutó un estudio de valoración contingente de tipo trasversal que evaluó la disponibilidad a pagar (DAP) en moneda nacional (Nuevo Sol Peruano, PEN, del 2017). Se seleccionaron los distritos de mayor población dentro del departamento de Piura, ubicado en la costa norte del Perú (20). Se estudió la población general $\geq 18$ años, proveniente de los distritos de Piura, Castilla y Tambo Grande, con ingreso o renta individual mayor que cero, y que estén dispuestos a pagar por tener acceso a la vacuna contra dengue. Como criterio de inclusión se consideró a cualquier individuo residente de los distritos de Piura, Castilla y Tambo Grande que acepte voluntariamente completar el cuestionario mediante entrevista personal. Se excluyeron del estudio a los sujetos que no hayan respondido la pregunta referente a la variable principal (DAP), quienes retiren su consentimiento de participación en la investigación, aquellos que refieran una DAP por la vacuna equivalente a cero real o dos veces mayor que su ingreso económico personal $(11,13)$, y turistas o personas que no sean provenientes del departamento de Piura.

La selección de participantes para la entrevista fue hecha por método de conveniencia directa en la plaza central de los distritos correspondientes $(11,20)$. Los individuos fueron invitados a participar por los entrevistadores a través de la firma de un consentimiento informado. Una vez aceptada su participación, se procedió a la aplicación del cuestionario en modalidad de entrevista personal como método de recolección de información.

La encuesta fue estructurada en tres secciones: 1) Información General y Sociodemográfica, 2) Conocimiento y Actitudes de Prevención contra el Dengue y 3) Disponibilidad a Pagar para una vacuna contra dengue. Las preguntas relacionadas a la disponibilidad a pagar tuvieron un formato binario-dicotómico doble de elección discreta, ya que este formato de pregunta ha mostrado resultados más sensibles y con menor sesgo $(12,21)$. El precio base inicial (S/. 170) se definió según en el precio de venta máximo de la vacuna disponible contra fiebre amarilla en Perú $(11,22)$ durante el año de investigación, y los intervalos de incremento se establecieron en S/. 40. Antes de cada sección, se hizo una lectura de las características básicas del dengue y la vacuna para uniformizar los conceptos relacionados a estas variables y la tecnología sanitaria. 
Este cuestionario fue desarrollado en base a criterios de conocimientos, actitudes y prácticas del dengue $(23,24,25)$, cuestionarios previos y estudios de valoración contingente ya ejecutados $(11,12,13,14,15)$.

La variable principal de la investigación fue la disponibilidad a pagar, definida como medida teórica de valoración monetaria de cantidad máxima que una persona está dispuesta a pagar para adquirir o tener acceso al servicio. En esta investigación, la DAP fue valorada según la moneda local Nuevo Sol Peruano del 2017 (S/. PEN 2017). Se tomaron como referencia las características de la única vacuna contra dengue comercialmente disponible hasta la fecha de ejecución de esta investigación: eficacia de 93,2\% para prevención de dengue severo, eficacia de $80,8 \%$ para reducción en hospitalización por dengue, y 65,6\% para la prevención de dengue sintomático virológicamente confirmado en mayores de 9 años (26).

Se incluyeron otras variables de carácter secundario para el análisis final, como nivel de educación, ser profesional de la salud, ser madre de familia, ingreso económico personal mensual, antecedente personal y familiar de dengue, conocimientos básicos sobre transmisión de dengue, hábitat del vector, cuadro clínico, potenciales complicaciones graves, haber comprado otras vacunas previamente, entre otras.

El cálculo de tamaño muestral se realizó usando la fórmula de Cochrane y las siguientes variables: un nivel de significancia $\alpha=0,05$, una potencia del $80 \%$ y una población de total de 997.000 habitantes en las regiones de estudio. El resultado final indicó una muestra mínima de 370 individuos $(27,28,29)$, quienes fueron estratificados proporcionalmente por tamaño poblacional de los distritos mencionados anteriormente: 199 (53,7\%) en Piura, 94 (25,5\%) en Castilla y 77 (20,9\%) en Tambo Grande.

Posterior a la creación de una base de datos mediante un proceso de doble digitación en el programa Microsoft Excel, se determinó un valor inicial de DAP a través de la mediana del valor máximo declarado por los sujetos del estudio. Se hizo una descripción de las variables sociodemográficas de interés y análisis exploratorio de los datos. Consiguientemente, se ejecutó un análisis estadístico de tipo no-paramétrico a través de un modelo logístico (Logit) para evaluar dicha DAP, y un modelo de regresión para evaluar factores asociados. Todos los análisis estadísticos se realizaron usando el programa Stata para Windows (Stata Corp, College Station, TX, EE. UU).

Todas las entrevistas se llevaron a cabo con previo consentimiento informado firmado por el sujeto. El investigador firmó un acuerdo de confidencialidad para mantener el anonimato y salvaguardar la información de los entrevistados. Esta investigación fue presentada (PI082-17) y aprobada (CEI/026-04-17) por el Comité de Ética de la Universidad Peruana de Ciencias Aplicadas (UPC) antes de su ejecución. 


\section{Resultados}

Se realizó un total de 396 entrevistas, de las cuales se perdieron 13 sujetos $(3,28 \%)$ por rechazo del consentimiento informado, 11 sujetos $(2,78 \%)$ por criterios de exclusión y 1 sujeto $(0,25 \%)$ por retiro del consentimiento informado. Tras las exclusiones, se logró tabular 371 cuestionarios válidos. Detalles de la distribución muestral se pueden ver en la figura 1.

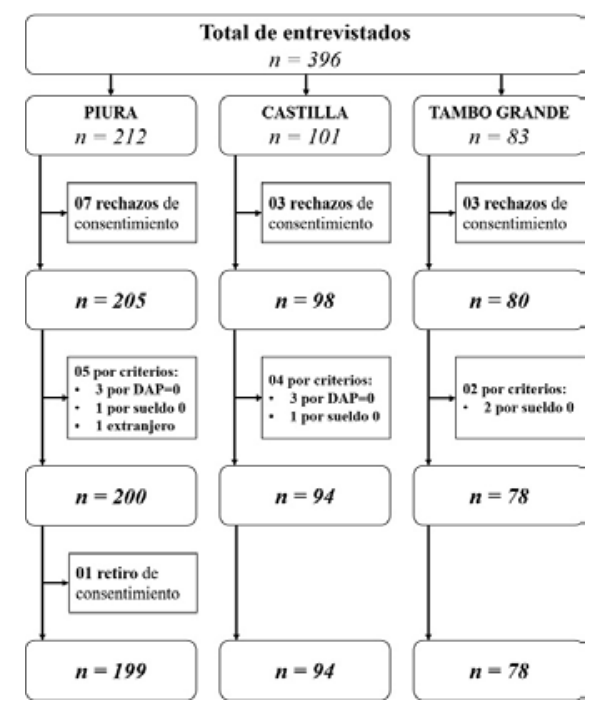

Figura 1.Distribución muestral de población entrevistada

Fuente: elaboración propia

De los cuestionarios válidos, la edad promedio de los sujetos era 42,1 $\pm 11,7$ años, 66,8\% era de sexo masculino, $47,4 \%$ eran casados, y solo el $24 \%$ tenía educación superior completa. 23 sujetos eran profesionales de la salud. 239 personas refirieron un antecedente personal de dengue, y 271 refirieron un antecedente familiar de dengue. El resto de las características poblacionales se pueden ver en las tablas 1 y 2 . 
El valor económico de la vacuna contra el dengue: un estudio de valoración contingente

Tabla 1.Características poblacionales de los sujetos entrevistados

\begin{tabular}{lrr}
\hline Variable & $n$ & $(\%)$ \\
\hline Edad $(\bar{x}, \pm)$ & 42,1 & 11,7 \\
\hline Sexo & 248 & 66,8 \\
Masculino & 123 & 33,2 \\
Femenino & & \\
\hline Estado Civil & 88 & 23,7 \\
Soltero & 176 & 47,4 \\
Casado & 107 & 28,9 \\
Otro & & \\
\hline Educación & 114 & 30,7 \\
Primaria Completa & 168 & 45,3 \\
Secundaria Completa & 89 & 24 \\
Superior Completa & & \\
\hline Profesional de la Salud & 23 & 6,2 \\
Si & 348 & 93,8 \\
No & & \\
\hline Madre de Familia & \\
Si & 103 & 83,7 \\
No & 20 & 16,3 \\
\hline Antecedente Personal de & & \\
Dengue & & \\
Si & 239 & 64,4 \\
No & 132 & 35,6 \\
\hline Antecedente Familiar de & & \\
Dengue & & \\
Sí & & \\
No & 271 & 73,1 \\
\hline Compra previa de vacuna & 100 & 26,9 \\
Si & & \\
No & 27,8 \\
\hline Población total de 123 mujeres & & 72,2 \\
\hline & & \\
\hline
\end{tabular}

Fuente: elaboración propia 
Tabla 2.Conocimiento relacionado a la enfermedad por dengue en los sujetos entrevistados

\begin{tabular}{lrr}
\hline Variable & $n$ & $(\%)$ \\
\hline Conocimiento sobre casos de dengue en su comunidad & 344 & 92,7 \\
Sí & 27 & 7,3 \\
No & & \\
\hline Conocimiento sobre transmisión de dengue & 291 & 78,4 \\
Sí & 80 & 21,6 \\
No & & \\
\hline Conocimiento sobre signos y síntomas del dengue ${ }^{\dagger}$ & 311 & 83,8 \\
Fiebre & 286 & 77,1 \\
Cefalea & 343 & 92,5 \\
Dolor óseo & 270 & 72,8 \\
Mialgia & 273 & 73,6 \\
Dolor retroocular & 145 & 39,1 \\
Malestar general & 92 & 24,8 \\
Dolor abdominal & 114 & 30,7 \\
Náuseas/vómitos & 102 & 27,5 \\
Sangrado & & \\
\hline Conocimiento sobre reproducción del vector & 188 & 50,7 \\
Aguas estancadas o similar & 183 & 49,3 \\
\hline Otras respuestas & & \\
\hline Conocimiento sobre posible gravedad del dengue & 222 & 59,8 \\
Grave, potencialmente mortal & 149 & 40,2 \\
\hline Otras respuestas & & \\
\hline † En base a respuesta sí/no de la encuesta & & \\
\hline
\end{tabular}

Fuente: elaboración propia

En términos de la DAP, el monto con mayor frecuencia de elección fue el de S/. 210 (22,37\%). El resto de DAP discretas tuvo frecuencias menores. Solo 1 participante refirió que pagaría más de las cantidades discretas incluidas en la encuesta. La distribución de frecuencias según la DAP se puede observar en el las figuras 2 y 3. La mediana también corresponde al valor de S/. 210 . Se hizo una distribución de los valores de DAP según tertiles de ingreso económico personal 
El valor económico de la vacuna contra el dengue: un estudio de valoración contingente

mensual en la figura 4, tanto para la mediana como para la media. Solo 6 sujetos percibían un ingreso económico menor del sueldo mínimo vital (30).

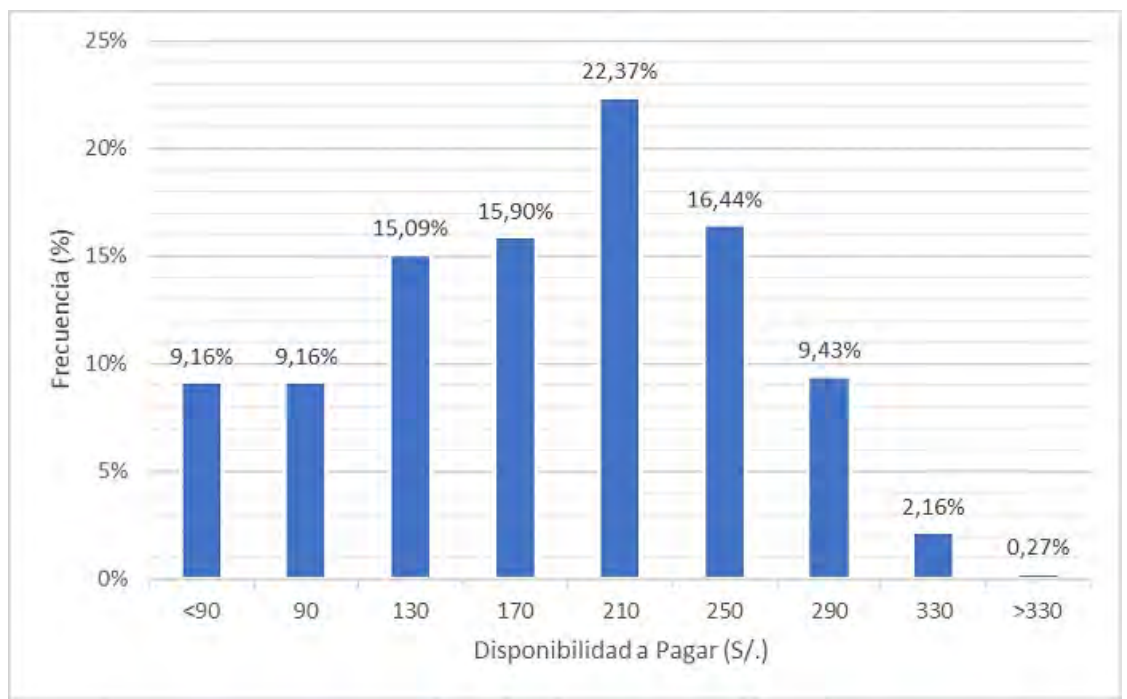

Figura 2. Frecuencia de máxima disponibilidad a pagar

Fuente: elaboración propia

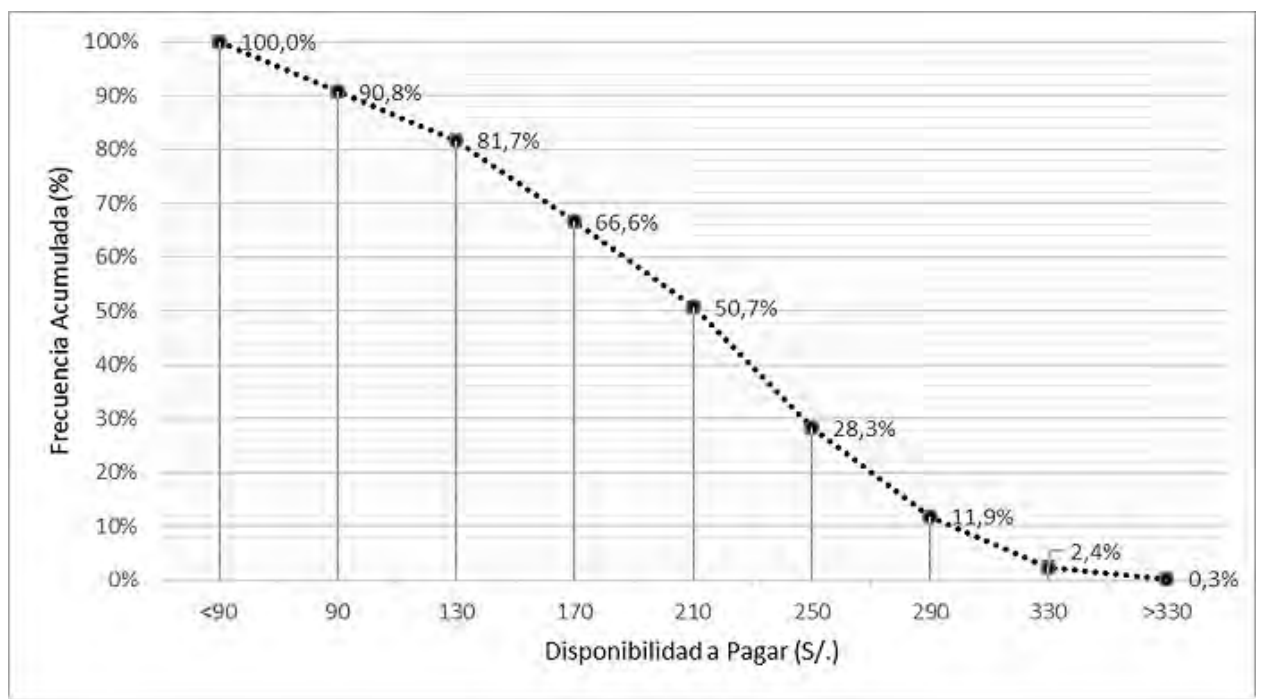

Figura 3.Frecuencia acumulada según disponibilidad a pagar

Fuente: elaboración propia 
Kocfa Chung-Delgado / Laura Navarro-Huaman

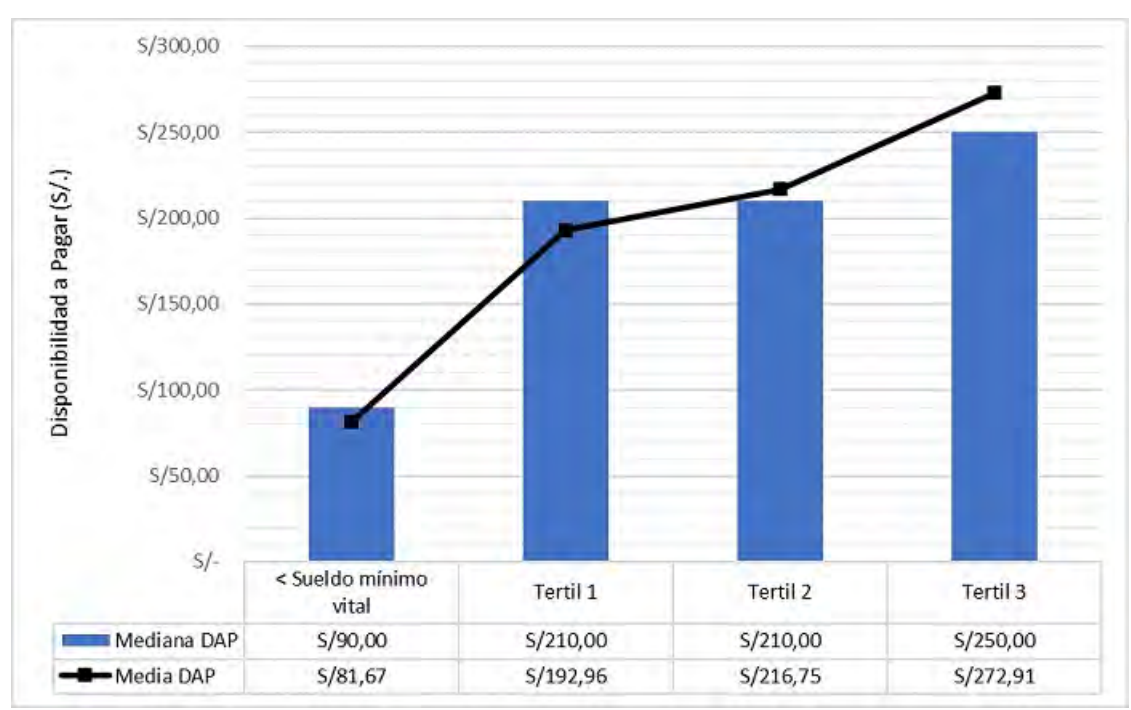

Figura 4. Disponibilidad a pagar según ingreso económico personal mensual

Fuente: elaboración propia

En el modelo Logit de regresión logística, se incluyeron las siguientes variables: edad, nivel de educación, si el sujeto es profesional de la salud, si es madre de familia, si tiene antecedente personal de dengue, si tiene antecedente familiar de dengue y si ha comprado vacunas anteriormente. La variable de precio tuvo un coeficiente de -0,009. Las variables que resultaron estadísticamente significativas para la compra de la vacuna contra dengue fueron ser profesional de la salud, nivel de educación, antecedente personal y familiar de dengue. Los coeficientes y valores $\mathrm{p}$ están descritos en la tabla 3. 
Tabla 3. Resultados del modelo Logit para disponibilidad a pagar

\begin{tabular}{lrr}
\hline Variable & Coeficiente & $p$-value \\
\hline Constante & 0,394 & Ref. \\
\hline Precio & $-0,001$ & 0,9 \\
\hline Edad & 0,007 & 0,29 \\
\hline Profesional de Salud & 0,241 & $<0,05$ \\
\hline Educación & 0,279 & $<0,05$ \\
\hline Madre de Familia $^{*}$ & 0,028 & 0,27 \\
\hline Antecedente Personal de Dengue & 0,455 & $<0,05$ \\
\hline Antecedente Familiar de Dengue & 0,296 & $<0,05$ \\
\hline Compra previa de vacuna & 0,062 & 0,13 \\
\hline
\end{tabular}

*Educación superior

Fuente: elaboración propia

\section{Discusión}

Con base en esta investigación se logra una aproximación a la valoración económica de una vacuna contra el dengue, desde la perspectiva de los consumidores.

Según los resultados, la disponibilidad a pagar mediana de los 371 cuestionarios válidos era de $\mathrm{S} / .210$, lo que indica que que el 50\% de los encuestados estaría dispuesto a pagar ese monto por tener acceso a la vacuna contra dengue. Cuando se compara este resultado con otros estudios, se puede observar que está por encima de los valores esperados. El estudio de Palanca-Tan (12) estimó que la mediana máxima de disponibilidad a pagar podía llegar a $\$ 60$, equivalentes a S/. 96,85 en Perú según la paridad de poder adquisitivo del Banco Mundial en el 2015 (31). Sin embargo, esta estimación fue hecha con base en el supuesto comparativo de que la vacuna cubriría 10 años de eficacia, algo que no se especificó en la presente investigación. El estudio de Lee et al. (13) presentó similares características de la vacuna: la mediana máxima fue de \$23,30 en Tailandia, equivalentes a S/. 37,61 en Perú (31). Otros estudios más recientes como el Harapan et al. (14) y Hadisoemarto y Castro (15) oscilan entre $\$ 1,94$ y $\$ 3,97$.

Se podrían conjeturar diversas hipótesis para explicar por qué los resultados de esta investigación se muestran por encima de las demás referencias. Lo principal e indispensable es que, en el tiempo que se desarrollaron las encuestas, la región de Piura estaba atravesando la peor epidemia de dengue registrada en la historia de Perú (6). En base a esta coyuntura, se espera un nivel de alarma y sensibilización mayor, que incrementa la utilidad esperada como 
valor social y médico a la vacuna. Por ende, esta no solo tiene la utilidad de prevenir el dengue, sino también un valor social agregado de poder detener la actual epidemia.

A pesar de ello, la distribución poblacional de la muestra en términos de DAP es muy similar al el resto de las investigaciones. Así como se ve en la figura 3, otros estudios muestran una distribución similar: mayor frecuencia en montos centrales (ejemplo: S/. 170 o S/. 210) y menor frecuencia en montos extremos. Es importante que se vea esta distribución de frecuencias, ya que muestra el comportamiento esperado de un individuo en el mercado (13). Según lo establece la teoría económica, a mayor precio menor la demanda esperada (15). En la figura 2 se puede apreciar que existe una distribución con ligero sesgo a la derecha (o sesgo positivo), y no una distribución simétrica, lo cual representa la decisión del consumidor de pagar lo menos posible por un bien o servicio. La misma distribución se ve en los estudios de Godoi et al. (11) y de Palanca-Tan (12).

De manera resaltante, hubo un sujeto entrevistado que reportó una DAP mayor a los valores discretos pautados en la encuesta. Este valor extremo se ve representado por el $0,27 \%$ y $0,3 \%$ en las figuras 2 y 3 , respectivamente.

Según lo esperado, el ingreso económico personal mensual tiene una aparente correlación con la DAP para la vacuna contra dengue: a mayor ingreso económico, mayor la DAP. Esta tendencia se puede evidenciar tanto para los valores de mediana, como media de DAP en la figura 4. Esta tendencia es la esperada, ya que otros estudios de valoración contingente identifican una correlación estadística entre DAP y el ingreso económico personal $(11,12,13)$. El estudio de Godoi et al. (11) correlaciona el ingreso económico y la DAP, y la identifica como la única variable asociada a la DAP estadísticamente significativa. Por otro lado, Palanca-Tan (12) encuentra esta misma tendencia y logra extrapolar esta asociación al ingreso económico familiar.

Con respecto al modelo Logit de disponibilidad a pagar, únicamente se identificó una variable con coeficiente negativo (-0,001): el precio. Esto implica que la relación entre precio y disponibilidad a pagar como elección es inversamente proporcional: a mayor el precio, menor la probabilidad de pagar por la vacuna. Este resultado va de acuerdo, tanto a la teoría económica como a otras evaluaciones de valoración contingente referente a la vacuna contra el dengue $(11,12,13,14,15)$. Una investigación realizada en Tailandia revela que, aunque suene lógica esta relación inversamente proporcional entre precio y un servicio de salud, no necesariamente es cierta ya que depende de muchos otros factores como la macroeconomía, acceso a servicios de salud e incluso percepción personal de salud (13). Según otra investigación de Indonesia, la disponibilidad a pagar tiene una relación con el precio asociado al estatus socioeconómico del entrevistado, el cual juega un rol importante a la hora de definir poder económico adquisitivo (15). 
Dentro del modelo se encontraron las siguientes variables como estadísticamente significativas para la compra de la vacuna: ser profesional de salud, tener educación superior completa, antecedente personal de dengue y antecedente familiar de dengue. El resto de las variables no fueron significativas.

La asociación con un alto nivel educativo es muy controversial en los estudios de valoración contingente. La investigación publicada por Hadisoemarto y Castro (15) reporta una asociación fuerte relacionada al nivel educativo del entrevistado, con mayor énfasis cuando la educación llega a nivel universitario. No obstante, otros estudios como el de Palanca-Tan (12) y Harapan et al. (14) hallan una asociación no estadísticamente significativa. A pesar de la variabilidad en resultados, sí se encuentra una tendencia positiva entre disponibilidad de pagar y nivel educativo, y ha sido descrita en la literatura científica (32). Esta correlación es similar, también, a la que se encuentra en estudios de valoración contingente asociados a otras vacunas como virus de papiloma humano (33).

El antecedente personal y familiar de dengue tiene una correlación evidente en este estudio, tanto como coeficiente y como análisis estadístico. Sin embargo, al igual que el nivel educativo, muestra resultados muy variables en la literatura ya publicada. Otros estudios de vacuna contra el dengue no asocian el antecedente personal de dengue con un incremento en la disponibilidad a comprar la vacuna $(11,12,14,15)$. Esto puede responder a la variabilidad de presentación clínica de la enfermedad, dependiente de aspectos como severidad del cuadro, días de enfermedad, necesidad de descanso médico o incluso cambios en niveles de calidad de vida y pérdida de años de vida ajustados por discapacidad.

Como fortaleza, esta investigación representa una de las primeras iniciativas en Perú en tratar de valorizar económicamente la DAP para una vacuna contra dengue. Información de este tipo es muy valiosa para las decisiones que se puedan tomar referente a esta endemia, y más aún si Perú continúa manifestando brotes epidémicos recurrentes. Pese a ello, la investigación también tiene limitaciones importantes, adicionales a las ya conocidas por el mismo método de valoración contingente $(34,35,36)$. En primer lugar, el tamaño muestral es reducido, lo cual limita las capacidades de interpretación y extrapolación hacia una población mayor. Piura es la región más endémica para dengue en Perú, pero el comportamiento epidemiológico de la enfermedad es muy variable a lo largo de todo el país. En segundo lugar, no se realizó un ajuste por variables en el modelo de regresión, lo cual limita la interpretación de los resultados iniciales. Así mismo, dado el reducido tamaño y complejidad de este estudio, no se contaba con suficientes datos para efectuar análisis de sensibilidad, como evaluaciones paramétricas, estimaciones de Turnbull y modelos de Kristrom, lo cual limita la validez externa del mismo. También se debe considerar el posible sesgo introducido por el investigador al ser ejecutada en modalidad de entrevista personal (36). 


\section{Conclusión}

La disponibilidad de pagar la vacuna contra el dengue aparenta estar sobreestimada en esta evaluación, pero los resultados son coherentes con la tendencia mostrada en la literatura. Esta investigación revela la necesidad de futuros estudios para definir y corroborar las conclusiones encontradas en el presente estudio: variar la DAP en base a dosis solicitadas y eficacia de la intervención, ajustar por acceso a servicios o seguros de salud, inclusión de comorbilidades, evidencia del mundo real para definir mejor el perfil de la vacuna e incluso abarcar más zonas geográficas para un análisis comparativo. Esta información farmacoeconómica será de gran utilidad para hacer evaluaciones más completas y científicas al momento de tomar decisiones sobre esta u otras vacunas contra dengue. En aras de la salud pública y de los objetivos de la lucha contra el dengue definidos por la OMS (2), se hace un llamado a las autoridades sanitarias y agentes reguladores a tomar en consideración la evidencia económica disponible referente a esta nueva tecnología para diseñar estrategias que garanticen la accesibilidad de la población a la misma.

\section{Referencias}

1. Sanofi Pasteur. Dengue Vaccine Registered in 16 Countries. 2016 [revisión 2017 jun. 03]. Disponible en: https://www.dengue.info/dengue-vaccine-registered-in-16-countries/

2. Organización Mundial de la Salud. Global Strategy for Dengue Prevention and Control 2012-2020. Ginebra: OMS 2012.

3. Guy B, Barrere B, Malinowski C, Saville M, Teyssou R, Lang J. From research to phase III: preclinical, industrial and clinical development of the Sanofi Pasteur tetravalent dengue vaccine. Vaccine. 2011;29:7229-41. https://www.doi.org/10.1016/j.vaccine.2011.06.094

4. Shepard DS, Coudeville L, Halasa YA, Zambrano B, Dayan GH. Economic Impact of Dengue Illness in the Americas. Am J Trop Med Hyg. 2011;84(2):200-207. https://www.doi.org/10.4269/ajtmh.20 11.10-0503

5. Shepard DS, Undurraga EA, Halasa YA. Economic and Disease Burden of Dengue in Southeast Asia. PLOS Negl Trop Dis. 2013;7(2):e2055. https://www.doi.org/10.1371/journal.pntd.0002055

6. Guzman J. Situación Epidemiológica de dengue en el Perú, a la SE 17-2017. MINSA. 2017; 26(17):540-543.

7. Centro Nacional de Epidemiología, Prevención y Control de Enfermedades. Casos de dengue según departamentos, Perú, a la SE 52-2017. Lima: Ministerio de Salud de Perú; 2017.

8. Organización Mundial de la Salud. Dengue Vaccine: WHO Position Paper - July 2016. Weekly Epidemiological Record. WHO. 2016;91(30):349-364. 
9. Roland D. Mexico Approves Sanofi's Dengue Vaccine but Pricing Questions Remain. The Wall Street Journal [Internet]. 2015. Disponible en: https://www.wsj.com/articles/mexico-approves-sanofis-den gue-vaccine-but-pricing-questions-remain-1449676613

10. McArthur MA, Sztein MB, Edelman R. Dengue vaccines: recent developments, ongoing challenges and current candidates. Expert Rev Vaccines. 2013; 12(8):933-953.

11. Godoi IP, Santos AS, Reis EA, Lemos LLP, Brandao CRM, Alvares J, et al. Consumer Willingness to Pay for Dengue vaccine (CYD-TDV, Dengvaxia ${ }^{\circledR}$ ) in brazil; Implications for Future Pricing Considerations. Front. Pharmacol. 2017;8(41). https://doi.org/10.3389/fphar.2017.00041

12. Palanca-Tan R. The demand for a dengue vaccine: a contingent valuation survey in Metro Manila. Vaccine. 2008;26:914-923. https://doi.org/10.1016/j.vaccine.2007.12.011

13. Lee JS, Mogasala V, Lim JK, Carabali M, Sirivichayakul C, Anh DD, et al. A Multi-country Study of the Household Willingness-to-Pay for Dengue Vaccines: Household Surveys in Vietnam, Thailand and Colombia. PLOS Negl Trop Dis. 2015;9(6):e0003810. https://doi.org/10.1371/journal.pntd.000 3810

14. Harapan H, Anwar S, Bustamam A, Radiansyah A, Angraini P, Fasli R, et al. Willingness to pay for a dengue vaccine and its associated determinants in Indonesia: a community-based, cross sectional survey in Aceh. Acta Tropica. 2017;166:249-256.

15. Hadisoemarto PF, Castro MC. Public acceptance and willingness-to-pay for a future dengue vaccine: a community-based survey in Bandung, Indonesia. PLOS Negl Trop Dis. 2013;7(9):e2427. https:// doi.org/10.1371/journal.pntd.0002427

16. Herdman M, Cole A, Hoyle CK, Coles V, Carroll S, Devlin N. Sources and Characteristics of Utility weights for Economic Evaluation of Pediatric Vaccines: A Systematic Review. Value Health. 2016;19:255-266.

17. Taylor RS, Drummong MF, Salkeld G, Sullivan SD. Inclusion of cost effectiveness in licensing requirements of new drugs: the fourth hurdle. BMJ. 2004;329(7472):972-975.

18. Organización Mundial de la Salud. Dengue Guidelines for diagnosis, treatment, prevention and control. Ginebra: OMS, 2010.

19. Plotkin S, Orenstein W, Offit P. Vaccines. Section One: General Aspects of Vaccination. 6.a ed. Filadelfia: Elsevier Saunders; 2013.

20. Perú, Instituto Nacional de Estadística e Informática. Encuesta Demográfica y de Salud Familiar ENDES. Lima: Instituto Nacional de Estadística e Informática, 2015. Cap. 1, Características de los hogares y la población; pp. 67-83.

21. Van der Pol M, Shiell A, Au F, Johnston D, Tough S. Convergent validity between a discrete choice experiment and a direct, open-ended method: comparison of preferred attribute levels and willingness to pay estimates. Soc Sci Med. 2008;67(12):2043-2050.

22. Perú, Dirección General de Medicamentos, Insumos y Drogas. Observatorio de Productos Farmacéuticos: vacuna antiamarílica. Dirección General de Medicamentos, Insumos y Drogas. Lima, Ministerio de Salud. 2016 [revisión 2017 abr. 02]. Disponible en: https://www.observatorio.digemi d.minsa.gob.pe/ 
23. Cáceres-Manrique FM, Vesga-Gómez C, Perea-Florez X, Ruitort M, Talbot Y. Conocimientos, Actitudes y Prácticas sobre Dengue en Dos Barrios de Bucaramanga, Colombia. Rev salud pública. 2009;11(1):27-38.

24. Cabrera R, Gómez de la Torre-Del Carpio A, Bocanegra Jesús AI, Correa Borit JM, Huamaní Fuente FJ, Urrunaga Poma PV, et al. Conocimientos, actitudes y prácticas sobre dengue en estudiantes de educación primaria en chorrillos, Lima, Perú. An Fac Med. 2016;77(2):129-135.

25. Hernández-Escolar J, Consuegra-Mayor C, Herazo-Beltrán Y. Conocimientos, actitudes y prácticas sobre dengue en un barrio de la ciudad de Cartagena de Indias. Rev Salud Pública. 2014;16(2):281-292.

26. Hadinegoro SR, Arredondo-García JL, Capeding MR, Deseda C, Chotpitayasunondh T, Dietze R, et al. Efficacy and Long-Term Safety of a Dengue Vaccine in Regions of Endemic Disease. NEJM. 2015;373(13):1195-1206.

27. Gunatilake H, Yang JC, Pattanayak S, Choe KA. Good Practices for Estimating Reliable Willingnessto-Pay Values in the Water Supply and Sanitation Sector. Manila: Banco Asiático de Desarrollo; 2007.

28. Fleischman-Foreit KG, Foreit JR. Willingness to pay surveys: for setting prices, for reproductive health products and services. A User's Manual. Population Council. Washington: USAID; 2004.

29. Accent RAND Europe. Review of Stated Preference and Willingness to Pay Methods. Accent RAND Europe. 2010.

30. Perú, Ministerio de Trabajo y Promoción del Empleo. Reumneración Mínima Vital, 2017. Lima: Ministerio de Trabajo y Promoción del Empleo; 2017. Disponible en: https://www.trabajo.gob.pe/

31. Banco Mundial. Price level ratio of PPP conversion factor (GDP) to market exchange rate. Washington: Banco Mundial; 2017. Disponible en: https://www.data.worldbank.org/

32. Filmer D, Pritchett L. The effect of household wealth on educational attainment: evidence from 35 countries. Popul Dev Rev. 1999;25(1):85-120.

33. Umeh IB, Nduka SO, Ekwunife OI. Mothers' willingness to pay for HPV vaccines in Anambra state, Nigeria: a cross sectional contingent valuation study. Cost Eff Resourc Alloc. 2016;14:8. https://do i.org/10.1186/s12962-016-0057-0

34. Cookson R. Willingness to pay methods in health care: a sceptical view. Health Econ. 2003;12:891-894.

35. Shono A, Kondo M, Ohmae H, Okubo I. Willingness to pay for public health services in rural Central Java, Indonesia: Methodological considerations when using the contingent valuation method Soc Sci Med. 2014;110:31-40.

36. Puig-Junoy J. Evaluación económica de medicamentos y tecnologías médicas. Avances. Barcelona: Universitat Pompeu Fabra; 2016. Cap. 2, El análisis coste-beneficio: métodos de estimación de la disponibilidad a pagar; pp. 57-75. 
El valor económico de la vacuna contra el dengue: un estudio de valoración contingente

Notas

* $\quad$ Artículo de investigación 